\title{
Prostatic Embryonal Rhabdomyosarcoma in A Five Year old Black Child From Nigerian North East: A Case Report And Review of Literature.
}

\author{
Annongu I $\mathrm{T}^{1}$,Mohammad $\mathrm{H}^{1}$, Babarinde $\mathrm{A} \mathrm{O}^{2}$, Wulfu Z B ${ }^{3}$,Mohammed $\mathrm{M} \mathrm{L}^{3}$ \\ Zarami A B ${ }^{4}$ \\ ${ }^{1,2}$ Department Of Radiology, Benue State University Teaching Hospital, Makurdi, Benue State. \\ ${ }^{3,4}$ Department Of Radiology, University Of Maiduguri Teaching Hospital, Maiduguri, Borno State.
}

\begin{abstract}
Soft tissue sarcomas are a heterogeneous group of tumours with a wide variety of presentation. Tumors arising from the soft tissues are uncommon in children, accounting for about $6 \%$ of all childhood malignancies. More than half (53\%) of these originate from the striated muscles and are called rhabdomyosarcomas (RMS) Almost two-thirds of RMS cases are diagnosed in children $<6$ years of age. They can arise at varied locations like the head and neck region, genitourinary tract, extremities, trunk and retroperitoneum.Rhabdomyosarcoma of the prostate in childhood however is rare more so in blacks. We therefore report a case of embryonal rhabdomyosarcoma of the prostate in a 5year old black child diagnosed by surgical pathology. On presentation, hischest radiograph showed evidence of metastatic chest disease. Patient had 7 courses of chemotherapypreceding prostatectomy which was done about 6months on presentation. Three months after the surgery, patient died of respiratory complications.
\end{abstract}

Keywords: Soft tissue sarcoma,embryonal rhabdomyosarcoma,prostate,black child.

\section{Introduction}

Rhabdomyosarcoma (RMS) is a sarcoma with skeletal muscle differentiation but which can occur in sites where skeletal muscle is not found suggesting that it is a tumor of primitive mesenchymal skeletal muscle differentiation. ${ }^{1}$ It is the most common soft tissue sarcoma in infants and children, with approximately 350 new cases in the United States each year and then third most common solid tumor in children after neuroblastoma and Wilms tumor, making up 10-15\% of all solid pediatric tumors. ${ }^{2}$ Rhabdomyosarcoma is also seen as the most common variety of the soft tissue sarcomas $(65.7 \%)$ in Jos Nigeria, ${ }^{3}$ similarfindingsalso documented by Brown et al. ${ }^{4}$ in Ibadan,south west Nigeria. It is believed to be the commonest form of soft tissue sarcoma in the first two decades of life. ${ }^{5,6}$

RMS incidence follows a bimodal age pattern depending on the histological subtype. The first window of increased incidence, which accounts for more than $50 \%$ of cases, occurs in the first decade. This period peaks at a median of 2 years of age, and consists mainly of embryonal (E-RMS) or botryoid RMS (B-RMS) subtype whereas the second peak occurs during adolescence and more commonly consists of alveolar RMS (A-RMS) subtype. $^{2}$ There is slight male predilection (M:F 1.67:1 $)^{7}$ with Caucasian children affected more often than other races. ${ }^{8}$ Sarcomas are relatively more common among children than in adults though among no age group are they common. Everyone who has been affected by the family of cancers known as sarcoma knows that it is rare. A recent publication about pediatric sarcoma, made it sound even rarer, stating that this aggressive cancerstrikes one child in a million, ${ }^{9}$ still another publication reemphasized; rhabdomyosarcoma of the prostate is extremely rare, especially in childhood. ${ }^{10}$ Here we report a case of prostatic RMS, anextremely rare childhood malignancy in a five old year black child in Nigeria, north east region.

\section{Case Report}

A 5 year old male primary school pupil who presented with one week history of difficulty and pains on passing urine, reduced urine volume and increased urinary frequency with terminal dribbling and anal protrusion especially after defecation. He is the last of 13 children by the same mother. One died at about a year after birth of febrile illness diagnosed as malaria. No family history of any congenital anomaly.

Patient has no known medical condition, has never been hospitalised nor received treatment in form of radiation nor an alkylating agent.His mother carried his pregnancy to term and was uneventful. No history suggestive of foetal macrosomia. She does not drink alcohol or smoke nor chew cigarette. On clinical examination, patient weigh was $14 \mathrm{~kg}$ which is $73 \%$ of his expected weight of $19 \mathrm{~kg}$ (underweight). He measured $103 \mathrm{~cm}$ in length with a Mid-upper arm circumference (MUAC) of $14.5 \mathrm{~cm}$. He had no birth mark.The head was normal in size, shape and symmetry and in normal proportion with the trunk. The facial settings were also normal in settings and symmetry.There was no kyphosis, lordosis nor scoliosis. The extremities and joints were 
symmetrical and essentially normal.Initial assessment of urinary tract infection (UTI) with haemorrhoids was made before investigations.Transabdominal ultrasound examination of the pelvis with a full urinary bladder using Logiq P5 sector scanner with a $3.5 \mathrm{MHz}$ transducer revealed a huge hetergenously fairly rounded irregular prostatic mass measuring $5.20 \mathrm{~cm} \times 5.50 \mathrm{~cm} \times 4.39 \mathrm{~cm}$ with a volume of $65.62 \mathrm{~cm} / 3$. No calcifications were noted within it. (Fig 1) There was slight increase in colour flow on Doppler interrogation.

The urinary bladder contained anechoic urine with regular and smooth walls. No feature suggestive of distal urethral obstruction was noted.Both kidneys were normal in position and outline with normal corticomedullary differentiation, however there were features of distal obstruction with mild calyceal dialation (grade II obstructive uropathy).No calculus was seen.Uretherocystography demonstrated a normal anterior urethral, the posterior urethral was thinned and stretched out but with smooth outline. There was free flow of contrast into the urinary bladder which was smooth with regular outline. No extravasation of contrast into the adjacent soft tissues was seen. No trabeculations nor sacculations noted. There was no bony erosions nor soft tissue irregularity noted in the pelvic region (Figs 2 and 3). On the chest radiograph there was left-sided superior mediastinal widening with convex outer border adjacent the medial border of the ipsilateral clavicle suggestive of paratracheallymphadenopathy. The remaining chest was essentially normal(Fig 4). The abdominal radiographs was also normal.

Computed tomography and Magnetic Resonance Imaging could not be carried out due to technical challenges so patients could not be investigated further with them for abdominal organs and lymphatic involvement.Assessment of a prostatic mass likely malignant was made, to be reviewed with histological outcome.The surgical pathology after tru-cut biopsy following radiological findings revealed Rhabdomyosarcoma of the prostate, the embryronal sub type on $\mathrm{H} \& \mathrm{E}$ stains and negative staining with desmin on Immunohistochemistry.(Figs 5a and b).Within five months on presentation, patient had received seven courses on cytotoxic drugs; Vincristine, Actinomycin, cyclophosphamide and other supportive drugs. He had Prostatectomy with suprapubic cystectomy and was discharged home in a stable condition.Patient was rushed back two months after discharge on account of respiratory symptoms and died shortly thereafter.

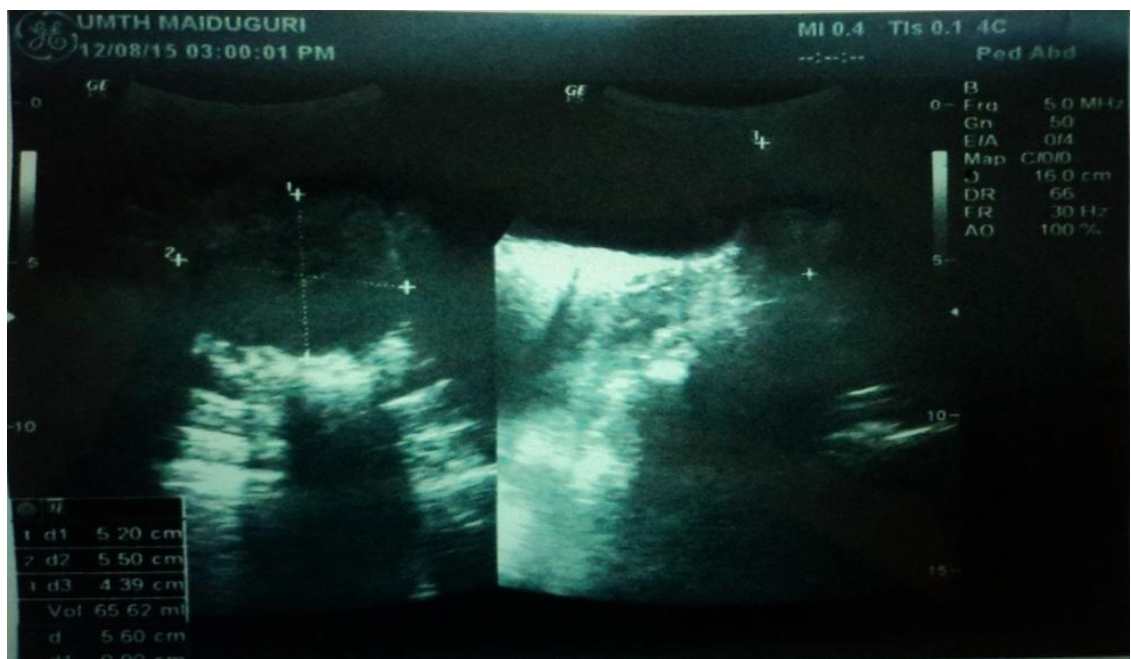

Fig.1 Pelvic ultrasound showing enlarged prostate with heterogenous echoes.

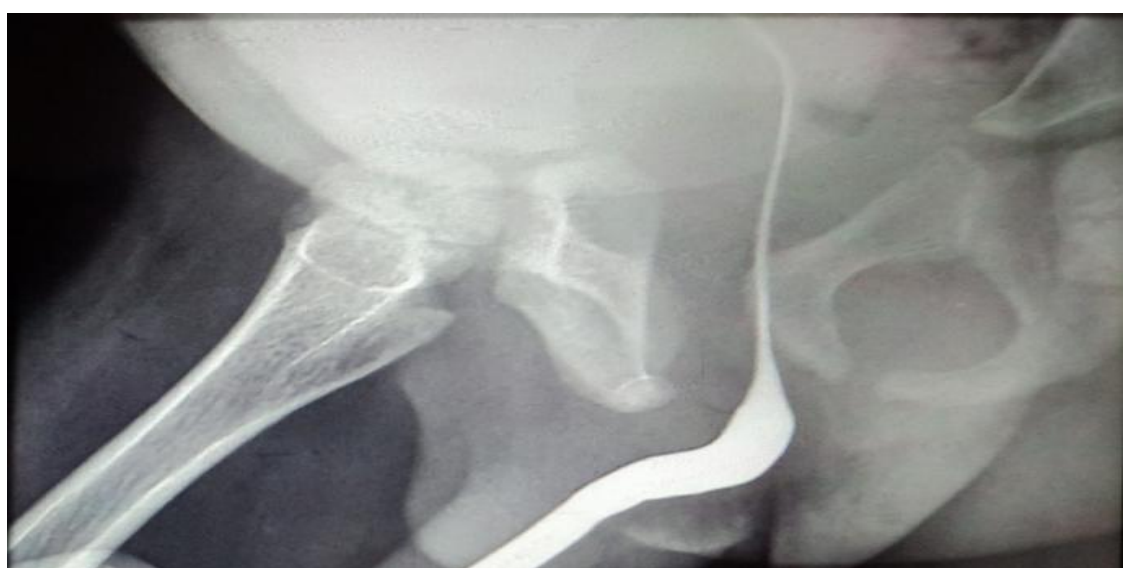

Fig.2 retrograde urethrography showing streatched-out posterior urethral. 


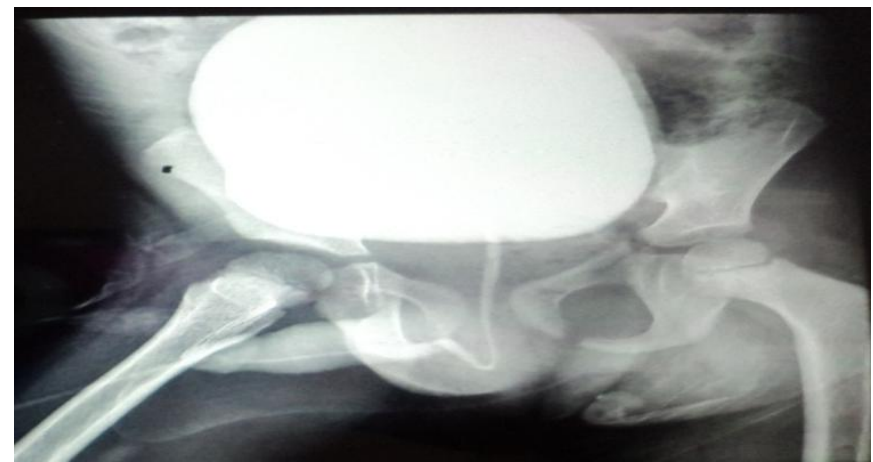

Fig.3 Cystography demonstrates a distended urinary bladder with smooth and regular wall.

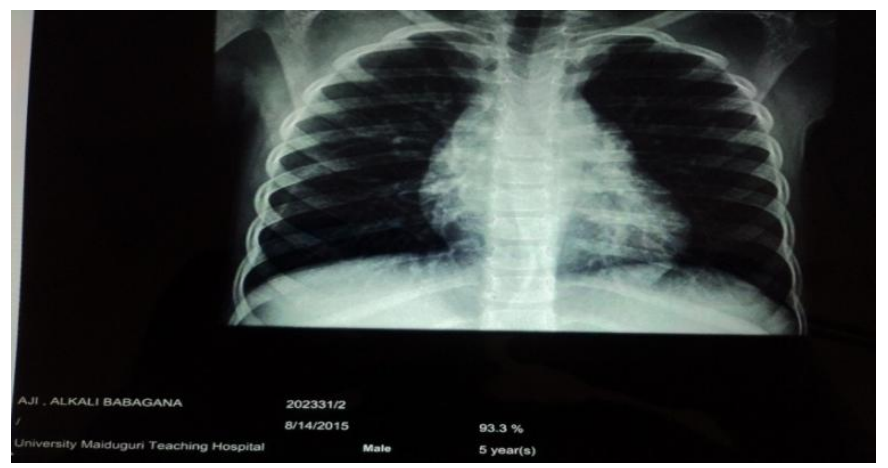

Fig.4 chest radiograph showing widening of the superior mediastenium suggestive of paratrachea lymphadenopathy.

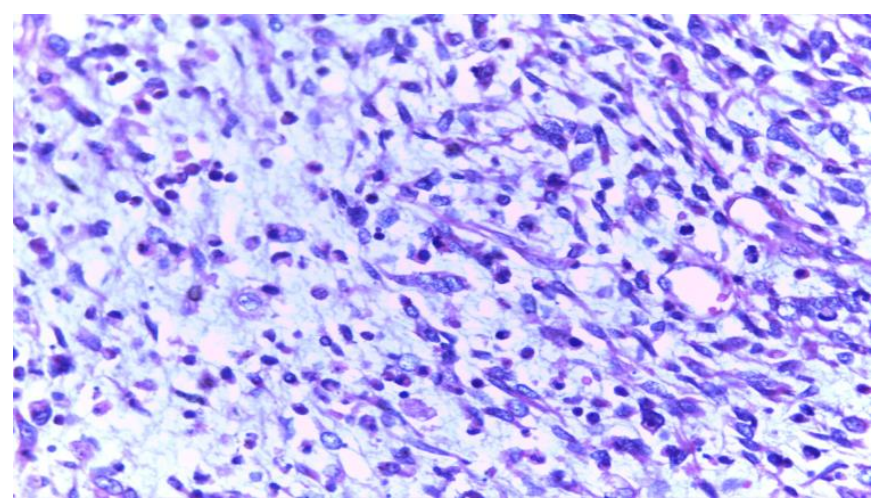

Fig.5a H\&E x40 stain showing primitive mesenchymal cells in various stages of myogenesis with small spindle to poorly differentiated round cells and scanty cytoplasm.

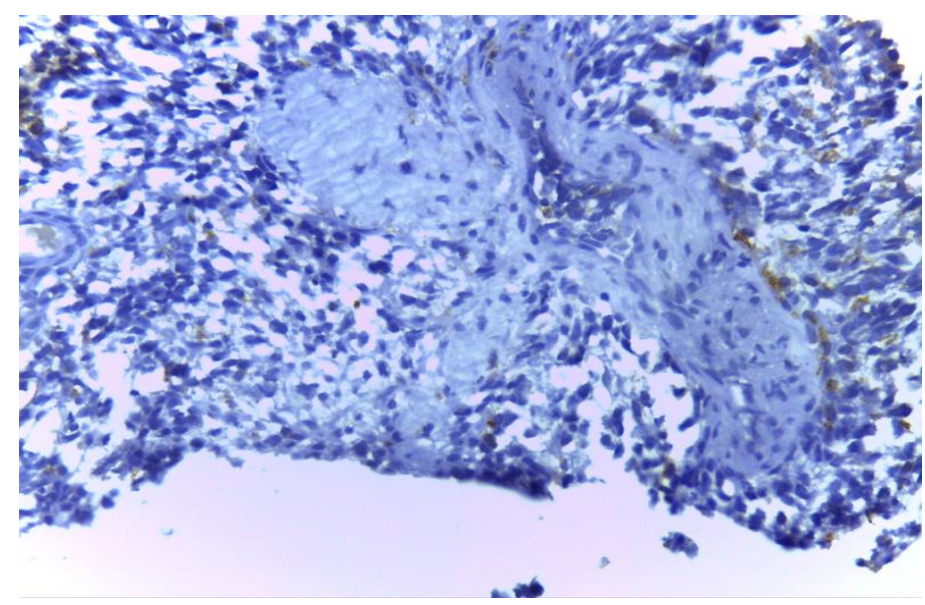

Fig.5b x40 IHC showing negative staining for desmin. 


\section{Discussion}

Though Pediatric sarcomas are rare, when they occur however, the embryonal subtype of Rhabdomyosarcoma (RMS) is the commonest variant. Rick et al demonstrated that this variant accounts for about $60-70 \%$ of cases. ${ }^{11}$ Similar finding was also shown by Agrons et al. ${ }^{12}$ These studies conform with the finding in our patient whom the histological report diagnosed embryonal subtype of the RMS. There are established conditions associated with developing of RMS, it can be part of a syndrome, ${ }^{13}$ but it can also be an isolated finding or occur in combination. However, given the young age of those affected; these are mostly congenital as opposed to acquired; ${ }^{14}$ these conditions could include:

\section{Syndromes}

1. Costello Syndrome. This manifests as delayed in growth, intellectual disability, loose folds of skin especially on the hands and feet, unusually flexible joint and abnormally prominent faecies characterized by a large mouth.

2. Gorlin's basal cell nevus syndrome. Patients with this syndromes present with multiple basal-cell cancer of the skin, rib/vertebral anomalies, intracranial calcifications, skeletal anomalies, distinctive facies-frontal and temporaparietal bossing, hyperterorism, and mandibural prominence.

3. Beckwith-Wiedemann syndrome. This is characterized by macroglossia, macrosomia, middle abdominal wall defects including Omphalocele, exomphalos, umblical hernias. Other features include ear creaseor ear pits, neonatal hypoglycemia and hepatoblastoma.

4. Fetal alcohol syndrome. Children with this syndrome are usually of short statues, low birth weight, small head size, poor co-ordination, low intelligence.

5. Trisomy 21 syndrome. Characterized by webbed neck, single palmar crease, short stature, lymphedema, broad cheast, low posterior hair line, low set ear amoung others.

\section{Genetic factors}

Two genetic conditions require particular mention: ${ }^{15,16}$

1. Neurofibromatosis is the most common syndrome in which RMS has been associated. Two types have been described: types 1 and 2. The criteria for the diagnosis of NF1 are met in an individual if 2 or more of the following signs are found:

-Six or more café au lait macules larger than $5 \mathrm{~mm}$ in the greatest diameter in prepubertal children and larger than $1.5 \mathrm{~cm}$ in postpubertal individuals

-Two or more neurofibromas of any type or 1 plexiform neurofibroma

-Multiple freckles (Crowe sign) in the axillary or inguinal region

-A distinctive osseous lesion, such as sphenoid dysplasia or thinning of long bone cortex, with or without pseudoarthrosis

- Optic glioma

-Two or more iris hamartomas (Lisch nodules) seen on slitlamp or biomicroscopy examination

-A first-degree relative (parent, sibling, offspring) with NF1, as diagnosed by using the above criteria.

\section{For NF2 Ferner et $\mathrm{al}^{17}$ give three sets of diagnostic criteria:}

1. Bilateral vestibular schwannoma (VS) or family history of NF2 plus Unilateral VS or any two of: meningioma, glioma, neurofibroma, schwannoma, posterior subcapsular lenticular opacities

2. Unilateral VS plus any two of meningioma, glioma, neurofibroma, schwannoma, posterior subcapsular lenticular opacities

3. Two or more meningioma plus unilateral VS or any two of glioma, schwannoma and cataract.

2. Li-Fraumeni Syndrome (LFS). Classic LFS is diagnosed when a person has all of the following criteria:

A sarcoma diagnosed before age 45 A first-degree relative with any cancer before age 45

A first-degree relative or second-degree relative with any cancer before age 45 or a sarcoma at any age.

Our index patient however, in both clinical evaluation and radiological investigations, no definitive links to any syndrome nor genetic factor were established.

\section{Environmental factors}

Environmental factors associated with RMS include radiation exposure and prior chemotherapy with alkylating agents. ${ }^{18}$ Our patient past medical history was uneventful with no history suggestive of exposure to radiation nor any chemical. Nearly $75 \%$ of GU-RMS cases are diagnosed before the age of 5 years. ${ }^{19}$ The age of diagnosis impacts prognosis independently from tumor histology. The diagnosis of the index case was established by surgical pathology. Immunohistochemistry with desmin was negative, some cells stain positively with desmin and all cells with vimentin. We do not however have the antibody for vimentin. Patients who are $<1$ 
year and $>10$ years old have a worse event-free survival rate compared to those between 1 and 9 years of age (53\% and $51 \%$, vs. $71 \%$, respectively). ${ }^{20}$

The indexpatient was within the good prognostic-favored age group of5years as at the time of diagnosis as well as the histological subtype as it has been shown that children with the E-RMS tends to survive beyond 5 year whereas those with the A-RMS subtype tends to metastasize early with a worse survival than E-RMS. ${ }^{21}$

However, tumour location was against him as shown that GU tumours of bladder/prostaticlocation have an unfavorable outcome. GU non-bladder/prostate (GU-NBP) sites, such as a paratesticular location (testes, epididymis and spermatic cord), vagina or uterus have better prognosis, and thus require less-intensive treatment. ${ }^{22}$ Krasdorf et al. ${ }^{7}$ documented thatunfortunately, up to $20 \%$ of patients have metastases at the time of diagnosis and based on tumour staging, tumor that has visibly spread to another "distant" part of the body is always Group IV.These are typically to lung and bone marrow. ${ }^{8}$ The index patient also demonstrated paratracheal lymphadenopathy at the time of presentation which conforms to the findings of 7 and 8 as documented.

\section{Conclusion}

Paediatric prostatic rhabdomyosarcoma is rare and usually has poor prognosis if there is metastasis, which is common way of presentation. Our index patient showed evidence of chest metastasis at presentation so that despite aggressive management patient latter died about 9months after presentation following chest complications.

\section{Refrences}

[1]. Crist W. Gehan E. Ragab A. et al. The Third Intergroup Rhabdomyosarcoma Study. J Clin Oncol.1995; 13: 610.

[2]. Dasgupta R. Rodeberg, D. Update on rhabdomyosarcoma. Semin Pediatr Surg.2012; 21: 68.

[3]. Ode M. Amupitan I. Misauno M. Mancha D. Onche I. Nwadiaro H.Soft Tissue Sarcoma.The Experience at JOS University Teaching Hospital. JOS Nigeria. IOSR-JDMS. 2015;14:47-49

[4]. Brown B. Oluwasola A. Childhood rhabdomyosarcoma in Ibadan, Nigeria: 1984-2003. Annals of tropical paediatrics. 2006; 26(4):349-55.

[5]. Missaoui N. Landolsi H. Jaidene L. Anjorin A. Abdelkader A. Yaacoubi M. et al. Pediatric rhabdomyosarcomas in Tunisia. Asian Pacific journal of cancer prevention: APJCP. 2010; 11(5):1325-7.

[6]. Hessissen L, Kanouni L, Kili A, Nachef MN, El Khorassani M, Benjaafar N, et al. Pediatric rhabdomyosarcoma in Morocco. Pediatric blood \& cancer. 2010; 54(1):25-8.

[7]. Kransdorf M. Murphey M. Imaging of soft tissue tumors. Lippincott Williams \& Wilkins. 2006; ISBN: 0781747716.

[8]. Rhabdomyosarcoma on WWW.radiopaedia.org/article/rhabdomyosarcoma

[9]. Joan D. How Rare Is Sarcoma Really? on http://sarcomaalliance.org/

[10]. Giuseppe M. Mario F. Massimo M. and Piero G. A Case of Prostatic Rhabdomyosarcoma in Childhood. Journal of Lower Genital Tract Disease, 2000; 4(3):172-174.

[11]. Rick R. Van R. Jim C. Johannes B. Foppe O. Kieran M. Johannes H.Imaging findings in noncraniofacial childhood rhabdomyosarcoma. Pediatr Radiol. 2008; 38(6): 617-634.

[12]. David M.Pathologic Classification of Rhabdomyosarcomas and Correlations with Molecular Studies. Mod Pathol 2001; 14(5):506514.

[13]. Malempati S. Hawkins D. Rhabdomyosarcoma: review of the Children's Oncology Group (COG) Soft-Tissue Sarcoma Committee experience and rationale for current COG studies. Pediatr Blood Cancer.2012; 59: 5-6.

[14]. Rhabdomyosarcoma on WWW.cancer.org

[15]. Sung L. Anderson J. Arndt C. et al.: Neurofibromatosis in children with Rhabdomyosarcoma: a report from the Intergroup Rhabdomyosarcoma study IV. J Pediatr.2004; 144: 666-667.

[16]. Malkin D. Li F. Strong L. et al. Germ line p53 mutations in a familial syndrome of breast cancer, sarcomas, and other neoplasms. Science.1990; 250: 1233-1234.

[17]. Ferner E. Susan M. Gareth R. Neurofibromatoses in clinical practice. Springer, 2011.

[18]. Ruymann F. Maddux H. Ragab A. et al.: Congenital anomalies associated with rhabdomyosarcoma: an autopsy study of 115 cases. A report from the Intergroup Rhabdomyosarcoma Study Committee (representing the Children's Cancer Study Group, the Pediatric Oncology Group, the United Kingdom Children's Cancer Study Group, and the Pediatric Intergroup Statistical Center). Med Pediatr Oncol.1988; 16: 33-34.

[19]. Ferrer F. Isakoff M. Koyle, M. Bladder/prostate rhabdomyosarcoma: past, present and future. J Urol. $2006 ; 176: 1283$.

[20]. Joshi D. Anderson J. Paidas C. et al.: Age is an independent prognostic factor in rhabdomyosarcoma: a report from the Soft Tissue Sarcoma Committee of the Children's Oncology Group. Pediatr Blood Cancer.2004; 42 : 64.

[21]. Meza J. Anderson J. Pappo A. et al.: Analysis of prognostic factors in patients with nonmetastatic rhabdomyosarcoma treated on intergroup rhabdomyosarcoma studies III and IV: the Children's Oncology Group. J Clin Oncol.2006; $24: 3844$.

[22]. Martelli H. Oberlin O. Rey A et al (1999) Conservative treatment for girls with nonmetastatic rhabdomyosarcoma of the genital tract: a report from the Study Committee of the International Society of Pediatric Oncology. J Clin Oncol 17:2117-2122. 